that a $p \mathrm{H}$ effect may underlie disappearance of zooplankton from "culturally enriched" waters of low buffering capacity. This disappearance would not only disrupt food chains leading to higher trophic levels (for example, fish), but would also remove predation on the algae and lead to further increases in algal density.

It would seem, though the authors do not suggest it, that if this effect is confirmed there would be circumstances in which $p \mathrm{H}$ monitoring with occasional slight manipulation could prove worthwhile "preventive medicine".

\section{LIQUID HELIUM \\ No a.c. Josephson Effect}

from a Correspondent

THE implication of some recent work by Musinski and Douglass (Phys. Rev. Lett., 23, 1541; 1972) is that many earlier reports which purported to describe investigations of the a.c. Josephson effect in liquid helium were in fact describing nothing of the kind. The a.c. Josephson effect is an important macroscopic quantum phenomenon predicted to occur in superfluids.

The only superfluids known are liquid ${ }^{4} \mathrm{He}$ and the electron gas in a superconducting metal, so there has naturally been a considerable incentive to compare these two systems: the analogues of phenomena observed in superconductors have been assiduously sought in liquid helium, and vice versa. $\mathrm{A}^{-}$basic feature of superfluids is that they display long range order, that is the motions of individual particles throughout a given volume of a superfluid are very highly correlated, by contrast with the random motions of classical kinetic theory. A particularly interesting situation arises when two such volumes are weakly coupled through a very narrow channel, so that neither perfect correlation between their particle motions, nor complete independence, can be assured. Josephson predicted that if there is also a difference in chemical potential (an energy per particle) between the two volumes, then periodic flow should occur in the connecting channel with a frequency proportional to the chemical potential difference. This remarkable assertion was soon verified experimentally for superconductors, and the phenomenon has subsequently been investigated in great detail. It was always anticipated that the effect would be much harder to find in helium (chiefly because helium atoms are neutral and much heavier than electrons), so the report by Richards and Anderson (Phys. Rev. Lett., 14, 540; 1965 ) that they had seen the a.c. Josephson effect in superfluid helium was hailed as a considerable scientific achievement.
Richards and Anderson inverted the experiment which had originally been proposed. They coupled two baths of helium through a narrow $\left(1.5 \times 10^{-3} \mathrm{~cm}\right)$ orifice, forced periodic flow within it by using a beam of ultrasound, and argued that anomalies should then be seen in the relative chemical potentials of the two baths. What they actually observed was that particular values of the difference in liquid level between the two baths, corresponding to differences in their chemical potentials, remained stable for long periods of time: liquid did not flow through the orifice to equalize the levels, as would be expected classically. The stable level differences were equally spaced from each other, in agreement with theory, and the effect ceased as soon as the ultrasonic transducer was switched off. After the experiment had been improved and repeated in several different laboratories the phenomenon seemed to be well authenticated, although, as Richards himself remarked in a later report, the stable states were always disturbingly hard to find.

Musinski and Douglass have now repeated the experiment yet again, but with two extra parameters at their disposal. They were able to create a small variable temperature difference between the two baths which, like a level difference, changes their relative chemical potentials. They also used an ultrasonic transducer whose frequency could be varied. If the values of level difference where arrest of flow occurred are really determined by the a.c. Josephson effect, then they should be changed by variation of either temperature difference or frequency. In the event, Musinski and Douglass found that the level differences were almost independent of temperature difference, and that their frequency dependence was in the wrong direction. Moreover, they also noticed that the stable states were occurring at particular absolute heights in one of the baths, and were not dependent on the level difference between the two baths. The inescapable conclusion is that the stable states of zero flow observed by Musinski and Douglass were not Josephson states, but artefacts arising from the experimental arrangements. It therefore seems highly probable that Richards and Anderson, and also the several other workers who came after them, misinterpreted their experimental data and thus did not, as they believed, observe the a.c. Josephson effect. If this is the case, it must come as a considerable disappointment to all scientists interested in superfluidity.

But why did earlier workers not apply the simple tests used by Musinski and Douglass to confirm the interpretation which they were placing on their data? Why has it taken seven years to show that their conclusions were probably erroneous? The answers

\title{
Background Radiation no longer Anomalous
}

FIVE years ago cosmologists were concerned to read in Physical Review Letters of the anomalously high values that emerged from the first far infrared measurements of the background radiation. These cast doubt on the role of the background radiation as a cornerstone in the evidence of a big-bang cosmology.

The cosmologists can now breathe again. In November last year the group responsible for the original measurements reported a repeat experiment in which no unusual flux was detected (Houck, Soifer, Harwit and Pipher, Astrophys. J. Lett., 178, L29; 1972), and next week's Nature Physical Science (January 22) contains a confirmatory report by a group at the Los Alamos Scientific Laboratory of the University of California.

The anomalous flux came to light in a rocket flight to discover whether the 2.7 K Planck curve describing the background radiation at radio wavelengths also applies in the submillimetre region where the blanketing effects of the atmosphere make observations difficult. It came as a surprise that the measurements showed a flux corresponding to a blackbody temperature between 4 and
$6 \mathrm{~K}$ higher than the expected $2.7 \mathrm{~K}$. Although it was almost immediately pointed out that this result is in conflict with some upper limits to the strength of the background radiation which can be obtained from studies of optical absorption lines in interstellar material, cosmologists will be relieved that rocket experiments are failing to reproduce the original findings.

The experiment carried out by the group responsible for the original result involved a telescope based on the original instrument, but with some modifications to rule out possible sources of interference which had been suggested to the authors, and the result was consistent with a $2.7 \mathrm{~K}$ background. This is also the result of the Los Alamos experiment, although in this case the analysis was complicated by problems encountered during the rocket flight.

There is still no explanation for the earlier anomalous flux, however. It now seems that it cannot have been of galactic or cosmic origin; the question is whether it was a consequence of some deficiency in the measuring instruments, or whether some phenomenon, possibly linked with the solar cycle, can be implicated. 